\title{
Le rôle de Maurice Dommanget dans la vie de la SER et des AHRF (1913-1976)
}

The role of Maurice Dommanget in the life of the SER and the AHRF (1911-1976)

\section{Serge Bianchi}

\section{Q OpenEdition \\ 12 Journals}

\section{Édition électronique}

URL : https://journals.openedition.org/ahrf/11083

DOI : $10.4000 /$ ahrf.11083

ISSN : 1952-403X

Éditeur :

Armand Colin, Société des études robespierristes

\section{Édition imprimée}

Date de publication : 1 septembre 2008

Pagination : 145-168

ISBN : 978-2-200-92515-4

ISSN : 0003-4436

Référence électronique

Serge Bianchi, "Le rôle de Maurice Dommanget dans la vie de la SER et des AHRF (1913-1976) ». Annales historiques de la Révolution française [En ligne], 353 | juillet-septembre 2008, mis en ligne le 01 septembre 2011, consulté le 01 juillet 2021. URL : http://journals.openedition.org/ahrf/11083 ; DOI : https://doi.org/10.4000/ahrf.11083

Ce document a été généré automatiquement le 1 juillet 2021.

Tous droits réservés 


\section{Le rôle de Maurice Dommanget dans la vie de la SER et des AHRF (1913-1976)}

The role of Maurice Dommanget in the life of the SER and the AHRF (1911-1976)

\section{Serge Bianchi}

1 Le paradoxe des liens entre Maurice Dommanget et la Société des études robespierristes mérite qu'on y réfléchisse un moment. L'un des auteurs et des collaborateurs les plus féconds de l'histoire de la Révolution française et de ses Annales historiques est aussi l'un des moins reconnus ${ }^{1}$ dans ce milieu, comme le montre le décalage entre l'hommage du monde syndical et politique à son décès, en 1976², et celui des Annales, essentiellement consacré à Albert Mathiez ${ }^{3}$, dont il fut longtemps le «bras droit ", au temps des pionniers! Est-ce lié au milieu professionnel, et au relatif isolement de l'instituteur de Morvillers? À des engagements politiques et syndicaux qui l'ont un moment éloigné (après 1926), sinon de la Révolution française, du moins des priorités de notre Société ? Nous développerons dans cette approche les liens forts et complexes qui ont uni un moment le fondateur - Albert Mathiez - et son disciple au temps de la Première Guerre mondiale, puis la fidélité de Maurice Dommanget au service des Annales et de notre Société, au cours d'un demi-siècle de contributions et de dévouement.

2 Trois axes seront ainsi abordés, après la brève évocation d'une carrière et d'une personnalité hors du commun ${ }^{4}$ :

-1. La place de Maurice Dommanget dans la Société.

-2. Le rôle joué dans les Annales.

-3. Les leçons de l'engagement d'un historien révolutionnaire. 


\section{Une vocation révolutionnaire précoce : le « prolétaire en veston ${ }^{5}$}

3 Nous pouvons rappeler quelques jalons de la vocation de l'historien révolutionnaire, expliquant son adhésion à la Société des études robespierristes dès $1913^{6}$. « Militant et pédagogue »: on ne saurait dissocier ces deux formes d'engagement dont l'osmose donne les clés de la carrière et de la personnalité de Maurice Dommanget.

Dès 15 ans, ce fils de boucher - non baptisé - dévore le Drapeau, périodique socialiste et anticlérical $^{7}$, et étudie Sylvain Maréchal à la Bibliothèque nationale ${ }^{8}$. Instituteur autodidacte, lecteur de la Révolution française d'Alphonse Aulard, il se syndique précocement, anime dès 19 ans une grève des études surveillées ${ }^{9}$ et écrit sous les pseudonymes de Jean Social et Jean Prolo dans la presse antimilitariste, bien que soldat en uniforme ${ }^{10}$ ou jeune instituteur ${ }^{11}$ ! Sanctionné pour un Manifeste des instituteurs syndiqués en $1912^{12}$, il est pourtant considéré comme irréprochable, et ce, pendant toute sa carrière, sur le plan professionnel. On le voit adhérent dès la première heure au parti socialiste unifié de Guesde et de Jaurès ${ }^{13}$. Il fonde le premier Syndicat de l'enseignement dans l'Oise le 11 juin 1914, à l'aube de la Grande Guerre ${ }^{14}$.

5 En tant qu'antimilitariste convaincu, il refuse l'Union sacrée pendant la guerre et participe aux réunions d'un groupe zimmerwaldien. En contact avec la minorité pacifiste - de Alphonse Merrheim, Pierre Monatte -, le sergent Dommanget est affecté à Beauvais pour raisons de santé, dans une compagnie de place. Paradoxalement, il se fait communiquer les archives de Beauvais pendant ses heures de garde et amasse, pendant la guerre, des matériaux pour une thèse sur la déchristianisation ${ }^{15}$. Il dénonce avec éclat le nationalisme cocardier de l'après-guerre dans des articles qui lui valent un sévère avertissement de l'inspection.

Il adhère dès 1920 à la III Internationale et collabore - épisodiquement - à L'Humanité. Il rejoint avec le syndicat des instituteurs de l'Oise ${ }^{16}$ la CGTU à sa création, et prend une influence croissante dans la Fédération unitaire de l'enseignement et à la rédaction de L'École émancipée ${ }^{17}$. Louis Bouët et Maurice Dommanget sont pressentis pour la rédaction de L'Humanité en 1924 pour succéder à Pierre Monatte et Alfred Rosmer ${ }^{18}$. Dommanget joue un rôle essentiel dans les congrès politiques et syndicaux (Grenoble, 1926). Il est élu à l'unanimité secrétaire national de la Fédération unitaire de l'enseignement de 1926 à 1928. Depuis le petit village de Morvillers où il reste instituteur, il assume une tâche harassante d'animation et de liaison. Mais cette responsabilité met en valeur les qualités fondamentales du syndicaliste, élu toutes tendances réunies, fait exceptionnel dans cette période : pendant ces deux années, 1500 nouveaux adhérents rejoignirent la FUN et lorsque Dommanget se retire, son bilan personnel est largement positif, même s'il entend s'effacer derrière un collectif.

7 À la croisée des chemins, on peut donner quelques éléments d'un portrait de l'orateur, du militant et du syndicaliste si présent jusque-là dans les combats robespierristes et révolutionnaires. Des qualités s'imposent: une éloquence vibrante, la volonté de rénovation pédagogique avec l'élaboration d'un manuel destiné aux masses, l'acharnement dans la défense des syndiqués face à la répression. Les contemporains ont été marqués par la puissance verbale de l'orateur, la force, le rayonnement qui se dégagent à la tribune dans l'affirmation des convictions du contestataire social et politique $^{19}$. "Quand Dommanget paraissait à la tribune, tout le monde n'avait qu'à se 
taire. Non seulement il connaissait à fond son sujet, mais il dégageait une autorité devant laquelle on ne pouvait que s'incliner. Sa voix grave, sa corpulence puissante, son évidente loyauté imposaient ses propos $»^{20}$.

8 Il entre alors, à quarante ans, dans une période de retrait relatif. Dès 1929-1930, il rompt avec le Parti communiste, sans rechercher une autre formation politique ${ }^{21}$, mais en gardant des contacts avec l'opposition à la ligne majoritaire du parti'22. En 1935 il assiste à la dissolution de la Fédération unitaire de l'enseignement, déchirée par les luttes de tendances depuis trois ans, puis sacrifiée sur l'autel de la réunification syndicale. Il est sanctionné à nouveau pour un discours antifasciste à l'automne 1935. Éloigné des postes de direction ${ }^{23}$, il peut dès lors se consacrer davantage à son œuvre d'historien, sans rien renier de ses convictions initiales, ce qui le conduit à refuser la Légion d'honneur en 1939, puis lui vaut d'être révoqué par le gouvernement de Vichy en $1940^{24}$. Au sortir de la guerre ${ }^{25}$, il participe à la reconstitution de la tendance syndicaliste de l'École émancipée. À partir de 1948 débute une retraite studieuse à Orryla-Ville. Le pédagogue cède la place à l'historien militant, celui de la Commune, du drapeau rouge, du $1^{\mathrm{er}}$ mai. Son bureau devient un véritable musée des contestations politiques et sociales ${ }^{26}$. En 1965, il reçoit ainsi des « jeunes » chercheurs et leur confi e, après une journée d'entretien, sa documentation sur le curé Meslier ${ }^{27}$. Pendant cette carrière si remplie, les liens de Maurice Dommanget avec la Société des études robespierristes ont été un moment très denses avant de se distendre, sans se rompre jamais.

\section{Le sociétaire des Études robespierristes (1913-1976)}

Maurice Dommanget s'est éveillé à la Révolution française autour de la personnalité de Sylvain Maréchal ${ }^{28}$, par la lecture de la Révolution française dirigée par Alphonse Aulard, de L'Histoire socialiste de Jaurès ${ }^{29}$ dans les années 1900, et des œuvres de Gabriel Deville ${ }^{30}$. La rencontre décisive est pourtant celle d'Albert Mathiez, «l'ardent historien de l'ardente histoire $»^{31}$, point de départ d'une amitié et d'une correspondance indéfectibles, jusqu'à la mort du maitre en 1932.

La première mention de son œuvre dans les Annales révolutionnaires est - en 1913 - une monographie sur la Révolution à Neuilly-saint-Front ${ }^{32}$. Dans une note de lecture, son directeur de mémoire, Albert Mathiez, multiplie les éloges, lui reprochant seulement de ne pas s'être "engagé plus hardiment dans la voie d'une histoire locale» $(\text { sic })^{33}$. Si Maurice Dommanget n'est pas présent à l'assemblée générale de 1913, il publie dans les Annales de cette année une étude sur Saint-Just ${ }^{34}$, ainsi que des notes sur des manuels préparant au certifi cat d'études ${ }^{35}$.

11 On peut dès lors suivre son assiduité dans les assemblées de la SER depuis la veille de la Grande Guerre ${ }^{36}$ jusqu'à son décès, survenu en 1976, à 88 ans.

12 Il est ainsi présent pour la $7^{\mathrm{e}}$ assemblée générale, qui compte - le 19 avril $1914-180$ sociétaires $^{37}$. Les liens de Dommanget et de Mathiez se renforcent alors, comme le montre le contenu de la revue, pour la réhabilitation de Robespierre et le dynamisme de la SER. Ce sont des liens politiques, intellectuels et humains traversés par quelques divergences. Celles-ci ne transparaîtront jamais dans la vie de la Société, ni dans les Annales révolutionnaires où la fi délité aux engagements du départ l'emporte sur tout le reste. 
13 Il est logique dans ces conditions que l'un des leitmotive des analyses de manuels par Dommanget - une quinzaine entre 1913 et $1918^{38}$ - soit le sort réservé à Robespierre par les manuels et l'opinion publique, dans la lignée de la création militante de la SER : « Robespierre est toujours offert en holocauste sur l'autel de la sacro-sainte Routine. On se demande quand fi nira ce petit jeu » écrit-il en 1914; et Mathiez répond en note: «Cela fi nira quand M. Aulard cessera de donner le La au diapason scolaire offi ciel ! " $^{39}$ Il milite alors pour que soit rendue à l'école «la justice de l'histoire » à l'égard de Robespierre comme des «rudes travailleurs $»^{40}$. En 1918, il s'écrie : « O ! Danton : pour essayer de te justifi er, que d'erreurs on commet en ton nom $»^{41}$. On appréciera ce tutoiement révolutionnaire, bien dans la ligne mathiezienne de l'époque...

À partir de 1915-1916, le rôle de Maurice Dommanget dans la Société devient prépondérant ${ }^{42}$, aux côtés d'Albert Mathiez. À l'assemblée générale de 1916, il traite en public « avec autorité, d'après des sources inédites, du mariage des prêtres à Beauvais pendant la Révolution $»^{43}$. Si la société comprend toujours 178 membres, elle le doit aussi à Maurice Dommanget, qui reçoit les "remerciements chaleureux» d'Albert Mathiez pour «le zèle couronné de succès avec lequel il a recruté de nouvelles adhésions à la Société $»^{44}$. En 1917, il participe à l'assemblée générale qui salue avec enthousiasme la victoire de la Douma russe contre le despotisme. Il partage l'essentiel des analyses de son "maître " sur les liens entre la Révolution française et le bolchévisme ${ }^{45}$. Le sergent Dommanget ${ }^{46}$, qui ne combat pas, contribue au maintien à flot de la Société, et publie sept articles dans la revue de "l'année trouble ${ }^{47}$ ! S'il n'est pas présent à l'assemblée générale de 1918, il s'engage à publier la première table des Annales. Il est alors omniprésent jusqu'en 1925, et ses responsabilités croissantes dans la Fédération unitaire de l'enseignement. Dommanget et Mathiez paraissent alors indissociables, tant pour le prosélytisme de la Société que pour le contenu de la revue.

De 1925 à 1949 pourtant, pendant un quart de siècle si important sur le plan politique et syndical ${ }^{48}$, il n'anime plus la Société, retranché à Morvillers, dans son école ${ }^{49}$ et sa bibliothèque, accaparé par des tâches syndicales et politiques de premier plan. Le décès d'Albert Mathiez en 1932 a probablement accentué ce relatif éloignement.

Mais d'autres raisons peuvent être brièvement rappelées. Unis par les combats syndicaux et politiques communs ${ }^{50}$, partageant la complicité des Annales et de la SER, les deux historiens sont séparés par leur milieu, voire des appréciations différentes des enjeux politiques et intellectuels des années 1920 comme des priorités de l'histoire révolutionnaire.

17 Certes, ils partagent des valeurs communes, très supérieures à leurs divergences. Mathiez, quoique brillant universitaire, soutiendra ainsi constamment le combat des instituteurs, la nécessité de manuels destinés aux enfants des travailleurs, comme le Manuel d'histoire de $1927^{51}$, qu'il est l'un des seuls universitaires de renom à recommander chaudement ${ }^{52}$. Les deux hommes adhèrent ensemble à la Troisième Internationale et au Parti communiste en 1920, et collaborent à l'Humanité dirigée par Amédée Dunois ${ }^{53}$ : "Depuis la guerre et surtout la révolution russe, une immense espérance a traversé la terre. Il faut préparer les esprits et les cœurs à la révolution par une propagande incessante et répudier l'opportunisme", écrit Dommanget en décembre 1920, partageant ainsi le combat de Mathiez. Ils prendront pourtant leurs distances avec Moscou progressivement, Mathiez dès 1923 $3^{54}$, Dommanget en 1929-1930. L'anticléricalisme commun des deux militants laïcs n'est pas pour rien dans la parenté des sujets traités, autour des cultes révolutionnaires ${ }^{55}$. Ils partagent une vision proche 
du mouvement social ${ }^{56}$. Dans ces domaines, Maurice Dommanget pourrait passer pour un disciple "fi dèle » de Mathiez. Il le défend d'ailleurs sur la question délicate de l'hébertisme en 1932, cautionnant la politique de Robespierre et de Saint-Just à son égard ${ }^{57}$. Il tente de concilier sa fidélité à Mathiez dans des appréciations sur « les hautes visées sociales » des robespierristes ${ }^{58}$, la dimension « libertaire » de la pensée de SaintJust $^{59}$, avec ses propres engagements, pourtant divergents ${ }^{60}$.

Si des distorsions nettes portent en effet sur l'appréciation de la Grande Guerre, où l'antimilitarisme viscéral de Dommanget s'oppose aux déclarations très " patriotiques » d'Albert Mathiez, elles se font "hors Annales». Sur un autre plan, le milieu des instituteurs de l'Oise ${ }^{61}$ cadre mal avec le milieu universitaire d'Albert Mathiez et de ses pairs, à Besançon comme à Paris. Dommanget se sent mal à l'aise au contact de certains " grands " professeurs ${ }^{62}$ : "J'ai fi ni par penser que je portais ombrage à toutes sortes de professeurs [...] quand ils écrivent des livres sur les mêmes sujets, ils me donnent un coup de chapeau en passant et ils me laissent tomber [...] Je suis bien plus connu à l'étranger qu'en France parce que je ne fais pas de jaloux [...] et puis d'ailleurs, je ne viens pratiquement plus à Paris $»^{63}$. Dommanget n'a jamais entrepris les recherches du doctorat qu'Albert Mathiez lui conseillait. Ce grand historien est resté « en marge de l'Université " ${ }^{64}$, s'il n'est pas « méconnu ». Après 1932, les positions internationalistes et libertaires de Maurice Dommanget - et ses publications nombreuses - se sont éloignées, un moment, du combat robespierriste originel ${ }^{65}$, sans jamais pourtant renier l'héritage et la mission générés par Albert Mathiez, et poursuivis jusqu'au décès de l'instituteur en $1976^{66}$. L'humour n'était d'ailleurs pas absent des relations entre les deux chercheurs : "L'historien de la terreur maniait souvent la parole et la plume à la façon du tranchant de la guillotine. Mais cette rudesse était plus apparente que réelle ${ }^{67}$.

19 Maurice Dommanget reprend une activité et une présence à la SER après le confl it mondial, et sa retraite studieuse en 1948. C'est bien lui qui rédige en 1958 un rapport sur l'histoire des 50 premières années de la SER, puis qui lance un Appel - en 1964 pour étendre son influence et la notoriété des Annales historiques de la Révolution française ! Cette fi délité d'un demi-siècle à une revue qui a tant compté pour lui mérite bien un hommage à la mesure des apports considérables du pionnier, du coopérateur infatigable d'Albert Mathiez.

\section{La place de Maurice Dommanget dans les Annales}

20 Nous voudrions d'emblée attirer l'attention sur un outil de travail dont le pionnier fut sans conteste Maurice Dommanget. C'est l'inventaire méthodique d'un siècle de publication des Annales, d'abord révolutionnaires, puis historiques de la Révolution française. Il permet de mesurer commodément et pour la première fois l'apport d'un historien à la revue, sur les plans quantitatif, thématique et quant au rayonnement ${ }^{68}$.

\section{Une contribution essentielle}

21 La base de données des Tables du centenaire, sous réserve de finalisation et de vérification, donne plus de 140 références directes à Maurice Dommanget dans les Annales $^{69}$. Il s'agit de 120 articles, documents ou glanes, de 1913 à 1968, et de 26 notices bibliographiques. Indépendamment de la qualité, on peut ainsi comparer cette contribution aux auteurs les plus «prolifiques », Albert Mathiez ${ }^{70}$, Georges Lefebvre ${ }^{71}$, 
Charles Vellay ${ }^{72}$, Gustave Laurent ${ }^{73}$, Gabriel Vauthier ${ }^{74}$, François Vermale ${ }^{75}$, Henri Sée ${ }^{76}$, Jacques Godechot ${ }^{77}$, Jean-René Suratteau ${ }^{78}$.

Si l'on met de côté l'apport écrasant du fondateur-pionnier Albert Mathiez - près de 700 références de la création à 1932, Dommanget se situerait probablement au deuxième rang des contributeurs, dans toute l'histoire des Annales. On peut cependant distinguer trois grandes périodes de contributions. De 1913 à 1924, il est omniprésent, signant entre 5 et 14 contributions par numéro, particulièrement au sujet des manuels scolaires du primaire. Dans certains numéros, il apparaît déjà comme le second d'Albert Mathiez, par le nombre d'articles publiés, avant d'autres rédacteurs, comme Gustave Laurent ou François Vermale.

De 1925 à 1949, il n'est mentionné que de façon épisodique, voire disparaît de certaines publications (1931-1938) pour des motifs qu'il conviendrait d'éclairer davantage, mais qui ont probablement trait à la charge du travail syndical et à des recherches menées hors du chantier de la Révolution.

Enfin, de 1950 à 1968, il reprend des contributions irrégulières dans les Annales historiques. On retrouve son nom dans les notes de lecture relatives à ses nombreux ouvrages concernant le mouvement social, le premier mai ${ }^{79}$ ou le drapeau rouge ${ }^{80}$, les hommes et les choses de la Commune ${ }^{81}$, les penseurs et les pédagogues socialistes. Il a fait ainsi l'objet de 26 notes de lecture, entre 1913 et $1966^{82}$.

Un tel bilan doit être complété par le contenu de cette coopération.

\section{Les centres d'intérêt}

Un survol de ces 50 années de contributions donne une répartition thématique sommaire. Les questions religieuses l'emportent largement depuis l'étude parue pendant la Première Guerre mondiale sur la Déchristianisation à Beauvais et dans l'Oise, jusqu'à la "débaptisation des enseignes à Beauvais » en 1962. Il la présente comme une chronique complète, retraçant le cheminement de sa thèse ${ }^{83}$. Il va jusqu'à s'excuser : «Je n'ai pas eu la prétention d'accaparer les colonnes des Annales révolutionnaires pendant plusieurs années $»^{84}$. Mais il obtient dès 1918 les compliments... d'Alphonse Aulard pour cet ouvrage contenant «une foule de faits curieux dans un récit très agréable à lire ${ }^{85}$. Les fêtes révolutionnaires, la toponymie, le calendrier, les changements de prénoms et de noms sont au cœur d'interrogations, centrées sur la laïcisation et la nature de la régénération. L'étude spécifi que de la déchristianisation mariage et abdications de prêtres, originalité des "curés rouges ${ }^{86}$, vandalismeiconoclasme ${ }^{87}$ - est moins présente que celle de ses formes "positives " ${ }^{88}$. L'ensemble (35 contributions) n'éclipse pourtant pas les autres thèmes : l'économie, la société, l'école.

Dans la ligne des recherches de son maître sur les subsistances et la vie chère, Maurice Dommanget s'est en effet intéressé aux questions de prix $^{89}$, de salaires, de mouvements sociaux, de moisson et de grèves de moissonneurs ${ }^{90}$, de ravitaillement, de maind'œuvre $^{91}$, de loyers, de biens nationaux. On peut rapprocher de ces thèmes les articles sur les sans-culottes et les Enragés ${ }^{92}$.

Le troisième thème dominant pour le pédagogue-citoyen est sans surprise celui de l'école et de l'enseignement de la Révolution ${ }^{93}$. D'où cette chronique de 14 manuels primaires, disséqués au regard de leur utilité et de leur efficacité. On peut en retirer 
l'idéal d'un manuel type qui permettrait une approche vivante et plurielle de la Révolution. Maurice Dommanget parle aussi des instituteurs républicains, anciens prêtres ou pédagogues civiques, comme des intermédiaires culturels nécessaires en temps de Révolution ${ }^{94}$.

Les préoccupations majeures, en dehors de ces dominantes, relèvent des grands personnages de la Révolution, penseurs politiques ou sociaux. Babeuf et le babouvisme ${ }^{95}$ s'imposent au moment du colloque de Stockholm, dans les années 1950-1960. Victor Daline le qualifie en 1963 de "meilleur connaisseur du babouvisme ${ }^{96}$. Puis sont étudiés les hébertistes, non sans préjugés sur le rôle joué dans la déchristianisation, Saint-Just ${ }^{97}$ et Robespierre, Sylvain Maréchal. Les portraits relèvent souvent de la réhabilitation, parallèlement à la nécessité de laisser les faits l'emporter sur les interprétations. Robespierre n'apparaît pourtant qu'à trois reprises dans des articles ${ }^{98}$, ce qui souligne une différence d'appréciation par rapport à Albert Mathiez, sans qu'on puisse y trouver matière à polémique, encore moins à rupture ${ }^{99}$.

Les autres apports relèvent de documents originaux et de glanes, concernant les centres d'intérêt cités précédemment.

\section{L'animateur des Annales}

31 Il est juste de rendre un hommage particulier à trois séquences de l'histoire désormais séculaire de notre revue. En 1919, Maurice Dommanget publie la première table (décennale) des Annales révolutionnaires, "organe de la Société des études robespierristes ", un volume de 109 pages remarquablement réalisé. Les thèmes, les auteurs et les statuts sont distingués par les types de caractères ${ }^{100}$ ou par la taille de la police ${ }^{101}$. On peut se rendre compte en le consultant de la tâche écrasante d'Albert Mathiez, qui occupe 13 pages sur $109^{102}$, et du rôle important de Dommanget, au gré de ses différentes contributions ${ }^{103}$. D'où l'hommage du secrétaire de la SER lors de l'assemblée générale du 8 avril $1920^{104}$ : «Pour terminer, j'adresserai les remerciements de la Société à Maurice Dommanget, l'auteur de la remarquable table décennale des Annales révolutionnaires. Nos lecteurs ont déjà pu apprécier cet instrument de travail qui a coûté à son auteur de longues heures d'effort et qui se signale par la sûreté des renseignements et l'esprit de méthode avec lequel il a été conçu. Nombreux sont les travailleurs qui l'utiliseront». Nous pourrions reprendre les conclusions d'Albert Mathiez: «En leur nom, j'adresse à Maurice Dommanget toute notre reconnaissance $~^{105}$. En 1919 comme en 2008, « ce répertoire ne sera pas seulement utile aux travailleurs. Il sera aussi notre meilleure justification parce qu'il montrera d'un coup d'œil aux plus prévenus l'étendue et la valeur de nos travaux $»^{106}$.

En 1958, c'est encore Dommanget qui dote la Société et les Annales d'un historique extrêmement précis, des origines à la fi $\mathrm{n}$ des années 1950 . Il y précise la rivalité entre Alphonse Aulard et Albert Mathiez en ces termes: "À la longue, un conflit devait surgir. Il était inscrit dans les choses prévisibles dès qu'apparaîtrait sur le plan de l'histoire révolutionnaire une "compétence" doublée d'un "caractère", un homme à la fois savant, indépendant, désintéressé et dynamique, susceptible de polariser autour de lui tous ceux qui, jusque-là, s'étaient courbés devant une "puissance" pour ainsi dire officielle. Cet homme fut Mathiez $»^{107}$. Il y explique aussi le regroupement, en 1927, des deux revues et la nomination des deux directeurs - Albert Mathiez et Gustave Laurent -, comme la progression discontinue du tirage et du lectorat, au gré des guerres et des 
joutes intellectuelles. On peut suivre dans son rapport, pratiquement année par année, l'évolution des adhérents, les difficultés fi nancières, la diffusion internationale de la revue. Il conclut sur un bilan mesuré et inscrit sur le papier les nécessités, «la voie dans laquelle la Société doit s'engager $»^{108}$ : afin d'augmenter le tirage et la diffusion par "l'action individuelle» (le recrutement des abonnés), la diffusion auprès des bibliothèques, des collègues et des sympathisants.

Il précise enfi n ces objectifs dans un appel vibrant lancé dans un numéro de 1964, sorte de testament pour les héritiers des Annales. Il y insiste sur "la nécessité plus impérieuse que jamais de faire connaître sérieusement la Révolution française "; " nous restons les seuls à représenter celle-ci »; « une revue quelle que soit sa facture, si remarquable qu'elle puisse être, ne peut vraiment progresser si elle ne fait pas l'objet d'une propagande et d'un recrutement systématique $»^{109}$.

$1919 ; 1958 ; 1964$ : ces simples jalons illustrent une présence à la fois utile et efficace... Cette action, étalée sur un demi-siècle, nous semble légitimer l'hommage qui lui est rendu aujourd'hui, dont nous n'avions pu nous acquitter pleinement dans notre première contribution aux Annales, consacrée en 1976 à... Maurice Dommanget, l'historien et l'homme.

\section{Les leçons d'un engagement}

"Le citoyen, le pédagogue, l'historien »: le triptyque du colloque de Beauvais de mai 1994 invite à synthétiser les leçons d'une carrière où la SER et les Annales tiennent une place considérable, mais non essentielle.

Cette vie droite ${ }^{110}$ et courageuse contient bien des leçons : le refus fondamental des contraintes hiérarchiques; le mépris de la répression ; le rejet du nationalisme. Surtout l'engagement total - pensée, action - au nom d'un idéal ; l'acharnement à concilier les tâches syndicales et pédagogiques; le refus de profiter, de parvenir qui fut la règle des syndicalistes révolutionnaires et que Dommanget a incarné au plus haut point. Reste le paradoxe initial : pourquoi Dommanget reste-t-il aussi peu connu dans la mémoire de notre Société et de notre revue?

\section{Un auteur « dépassé »?}

Tout passe, même une façon d'écrire l'histoire. Nous avons déjà formulé111 quelques réserves sur l'interprétation des écrits de Dommanget, à la lumière d'acquis et de méthodes récents de la recherche historique. L'historien lui-même n'aurait pas apprécié un bilan trop élogieux d'une œuvre qu'il remettait sans cesse en question.

Une histoire trop «militante»? Les engagements radicaux de Maurice Dommanget transparaissent-ils dans les Annales? On peut avoir certaines gênes rétrospectives dans les parallèles entre la période du salut public et le bolchévisme de 1918-1919 ${ }^{112}$. Mais les analyses de l'auteur reposent d'abord sur une documentation authentique. Les lectures sont toujours amples. Dommanget rend ainsi hommage à Hyppolite Taine, comme à Alphonse Aulard en 1958, malgré les polémiques passées avec des écoles ou des auteurs dont il conteste les analyses. On ne retrouve d'ailleurs pas dans les Annales certains des jugements enflammés ${ }^{113}$ des Éditions ouvrières (et de Spartacus), destinés à un public plus ciblé et militant, comme: "Babeuf n'est pas mort en vain en martyr. Sa 
conjuration est l'origine même du mouvement prolétarien et révolutionnaire moderne "114. Ses idéaux de "révolution permanente", de chaîne des révoltes (paysannes) ne se révèlent guère dans ses articles «scientifi -ques ». Ce dédoublement de l'écriture, cette retenue expliquent sans doute l'estime qui a entouré la parution de ses maîtres livres.

Des concepts « discutables »? Dans les années 1913-1922, Dommanget est marqué par les conditions et les priorités de la parution des Annales révolutionnaires et celles de son maître, Albert Mathiez. Il partage le souci de la défense de l'Incorruptible, contre vents et marées. Il ne pousse pas à leur terme la définition théorique de certains concepts, comme ceux de sans-culotterie ou de "curés rouges ", alors que sa culture politique l'y disposerait. Il se range du côté des conceptions de Mathiez, "l'historien le plus averti de la question religieuse sous la Révolution $»^{115}$, sur les cultes révolutionnaires (contre Alphonse Aulard, tenant de leur laïcisation) alors que ces notions ont été bien précisées depuis ${ }^{116}$, et que Dommanget a pris des distances dans d'autres publications, sur la laïcité ou la libre-pensée par exemple.

Nous pouvons contester aussi vigoureusement une assertion de 1917 relative au " vandalisme » : « La sombre rage qui poussa des sans-culottes quelles que fussent leurs tendances à renverser, gratter, piétiner, sabrer, briser, pulvériser ou jeter à la voirie tous les talismans que la religion proscrite exposait à la vénération des fidèles $»^{117}$. Les études récentes sur le «vandalisme» révolutionnaire - qualifié par ailleurs d'iconoclasme ${ }^{118}$ - auraient dû inciter à plus de prudence. Il en est de même sur la pratique du calendrier républicain, à notre sens.

41 Enfin, on peut critiquer la parution de fragments successifs, certes complémentaires, de sa thèse, le conduisant à se justifier d'accaparer les Annales. Les articles se suivent sans mise en perspective de leur spécificité. La pratique d'un comité de lecture rend aujourd'hui dépassées de telles pratiques. L'auteur était parfaitement conscient des limites de ses analyses: "J'en ai assez dit pour m'excuser et pour donner à d'autres l'idée de pousser plus loin les investigations $»^{119}$.

Ces réserves faites, les apports de Maurice Dommanget demeurent considérables, voire essentiels. Dans bien des domaines en effet, ceux-là même sur lesquels ont porté nos réserves, il a joué le rôle d'un accoucheur de l'histoire.

\section{Les vertus de l'histoire locale}

43 Jacques Bernet ${ }^{120}$ et les historiens de l'Oise et de la Champagne ont rendu en 1994 un vibrant hommage à Dommanget, historien de terrain. La déchristianisation à Beauvais et dans l'Oise ${ }^{121}$ a nourri nos propres études sur celle de la région de Corbeil ${ }^{122}$. Paradoxalement, Albert Mathiez encourageait Dommanget à insister sur la monographie en 1913, alors que ce dernier passe aujourd'hui pour le type même de l'historien local. Les portraits des "curés rouges " (Sallentin et Floury), des députés, des représentants en mission, les archives des sociétés populaires et des comités de surveillance, les séries de prix et de salaires: rien n'échappe à la documentation « locale » de l'historien, ancré sur son terroir et sur des Archives départementales qu'il a écumées pendant la Grande Guerre. Au reste, près de la moitié de ses contributions se situent dans l'espace oisien. Dommanget fut bien un historien de terrain, un défricheur inlassable dont les œuvres éparses mériteraient d'être plus accessibles ${ }^{123}$, pour la culture des enseignants locaux. Il se situe aussi dans la ligne d'un Bicentenaire de 1789 
qui a marqué souvent le renouveau de l'histoire locale, renforçant les liens entre les chercheurs universitaires et les groupes de recherches ou sociétés savantes enracinés dans la vie de leurs cités ${ }^{124}$.

\section{La vulgarisation pédagogique}

La chronique des manuels scolaires de l'école primaire a cessé dès 1920. Elle est pourtant révélatrice d'une des priorités de Dommanget: vérifier comment est enseignée l'histoire de la Révolution aux jeunes. Il dissocie souvent le contenu idéologique des qualités pédagogiques, louant « l'esprit vraiment laïc et républicain » d'un manuel, par ailleurs « inexact " pour " tout ce qui se rattache au socialisme ${ }^{125}$. Il traque l'érudition inutile: "L'abus des noms propres déshonore la pédagogie » ${ }^{126}$, l'absence de définition des termes ${ }^{127}$, comme les lacunes des manuels sur l'histoire sociale, la déchristianisation, le babouvisme ou la garde nationale, les biens nationaux. Il défi nit une véritable charte de l'auteur de manuels dès 1917: " On ne saurait trop étendre chez l'élève, à plus forte raison chez l'instituteur, l'esprit d'investigation et de recherche, le souci de la vérité. Rien ne développe mieux l'esprit critique et la controverse ${ }^{128}$, rien ne fixe mieux les connaissances que cette pratique des lectures nombreuses et contradictoires $»^{129}$. L'idéal est d'œuvrer pour "ces rudes travailleurs qui ont fait la France moderne et qui, pour prix de leur souffrance, de leurs sacrifices, attendent toujours en vain la justice de l'histoire $\aleph^{130}$. Il nous semble que les préoccupations pédagogiques auraient aujourd'hui leur place, à bien redéfinir, dans notre revue ${ }^{131}$. Maurice Dommanget fut partie prenante dans un manuel destiné aux élèves du cours moyen, lancé en 1927 par la Fédération de l'enseignement, qu'il dirige, qui privilégie l'histoire « du travail et de la civilisation, celle du peuple, des métiers, du progrès humain " par rapport à « la politique, la diplomatie, l'histoire des rois et des guerres $»^{132}$. Rarement la pédagogie active ${ }^{133}$ et l'histoire des contestations ont été aussi étroitement unies que dans l'œuvre de Dommanget, ce " prolétaire en veston ». Entre l'idée de base : «La lutte des classes traverse l'école» et la vocation de l'instituteur : "Je ne veux être que maître d'école ", Maurice Dommanget a pourtant privilégié dans sa pratique la pédagogie sur le militantisme, offrant à ses élèves «des lectures nombreuses et contradictoires $»^{134}$.

\section{Le prosélytisme pour les Annales de la Révolution}

De 1913 à 1964, Maurice Dommanget a affiché la volonté de démultiplier l'audience de la Société et la diffusion des Annales. Même si le premier état publié des abonnements est relativement modeste pour l'Oise, il est félicité par Mathiez pour avoir maintenu le niveau des adhérents pendant la Grande Guerre. Son appel publié en 1964 reste d'une grande actualité, au moment du centenaire de notre Société et de notre revue, à propos d'un tirage qu'il juge certes convenable mais « insuffisant $»^{135}$. Il vise les instituteurs, les « républicains modérés » et les « formations d'avant-garde, les centres universitaires, la diffusion dans les revues proches $» . .$.

Mesurant le chemin parcouru en un demi-siècle, il se félicite de l'audience internationale et universitaire croissante des Annales à la fin des années 1950, «le rayonnement de la revue sur le plan mondial ", mais appelle à une consolidation financière. Surtout il estime que le combat robespierriste est mal engagé et que depuis 
1945, « la cause de l'Incorruptible a perdu une partie du terrain gagné de haute lutte ", et à travers lui « la cause de la Révolution française en particulier et des révolutions en général ».

Nous voudrions insister sur ce qui nous identifie au combat commun de Mathiez et de Dommanget dans les années 1910-1920. Nous ne partageons pas tous leurs visions respectives des rôles joués par Robespierre et les robespierristes dans l'histoire politique et sociale de la Révolution. Mais, à travers la réflexion sur la période du Comité de Salut public, de l'économie dirigée et de la "révolution culturelle », c'est l'ensemble de la Révolution et de ses caractères originaux que nous entendons prendre en compte dans la Société des études robespierristes et dans les pages des Annales historiques, en respectant un héritage scientifique considérable et désormais séculaire, sans nous focaliser sur les étiquettes, agissant ainsi comme Dommanget l'a fait toute sa vie, au-delà de réelles divergences d'appréciations ${ }^{136}$ avec Albert Mathiez ou d'autres membres de la Société.

Il éclaire ainsi de la façon la plus honnête le débat permanent de l'adéquation originelle entre robespierrisme et Révolution française, quand il précise en 1958 la voie «dans laquelle la Société doit s'engager». "Elle doit continuer l'étude des faits, des institutions et des hommes de la Révolution en s'élevant bien au-dessus des querelles de factions, en utilisant toutes les méthodes d'investigation et de recherches $»^{137}$. Qui ne souscrirait à l'ensemble de la proposition, définissant le socle de notre identité ?

Pour conclure, qu'il nous soit permis d'affirmer notre dette intellectuelle et morale envers ces apports de Maurice Dommanget, et de partager les conclusions de celui qui a tant œuvré pour notre Société et notre revue. Dans son vibrant Appel de 1964, le bras droit d'Albert Mathiez dans les années 1915-1925, l'un des grands pionniers de notre Société, nous invite à nous dépasser : "De toute façon, il faut agir. Nous ne pouvons plus piétiner longtemps ${ }^{138}$. Nous pouvons imaginer le lutteur énergique, traçant à la tribune les missions des membres de la Société : «Chacun de nous, dans son entourage, peut et doit trouver des abonnés ou cotisants nouveaux. Il ne faut pas croire que la prospection se limite aux professeurs ou amateurs d'histoire». Afin d'entretenir la flamme ${ }^{139}$, nous pourrons sans doute méditer la leçon ultime du "prolétaire en veston »: "La société doit s'efforcer de gagner une base populaire, ce qui ne peut être obtenu que par un effort spécifique de recrutement, le développement de la partie de la revue réservée à la chronique, la pénétration dans les groupements démocratiques et les périodiques de vulgarisation d'histoire $»^{140}$ !

\section{ANNEXES}




\section{Maurice Dommanget et sa classe à Morvillers, 1913}

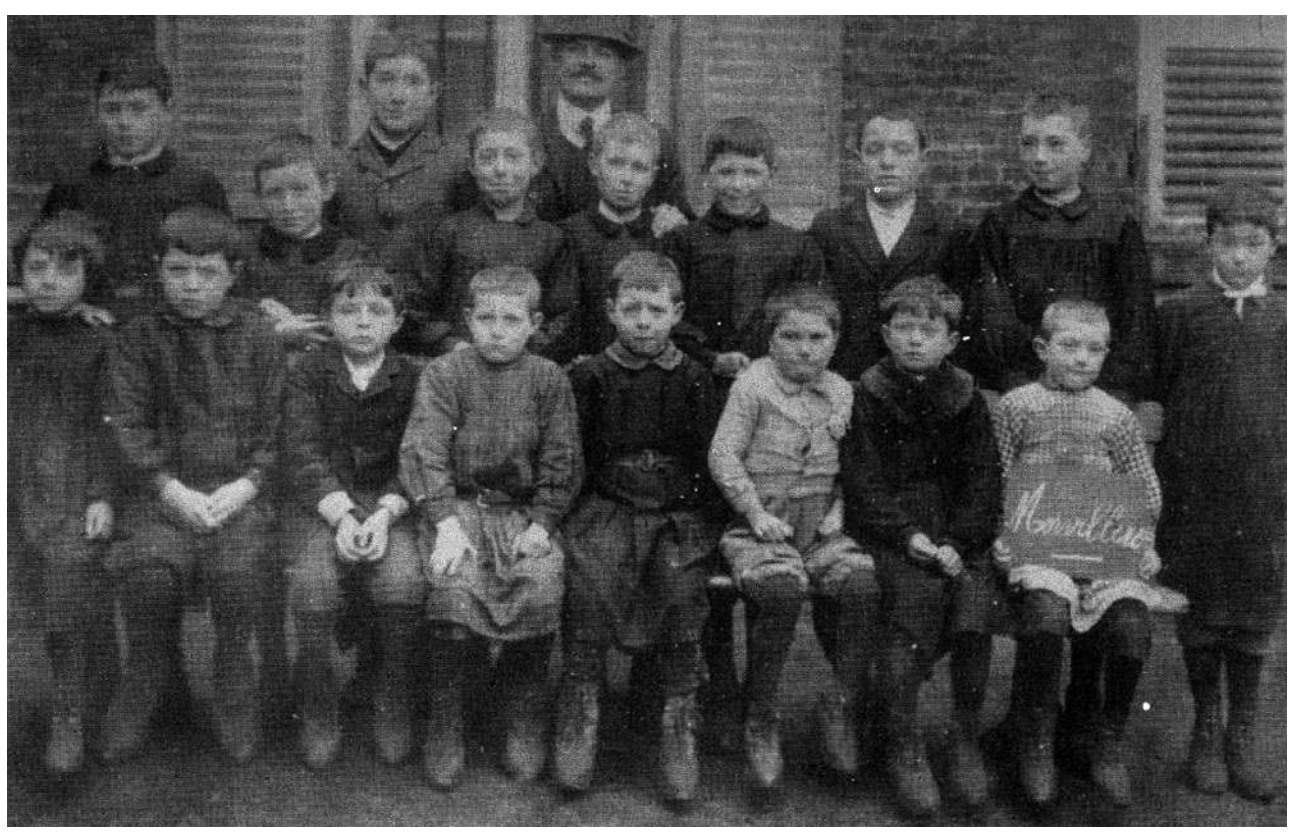

\section{Congrès de Grenoble, 1926}

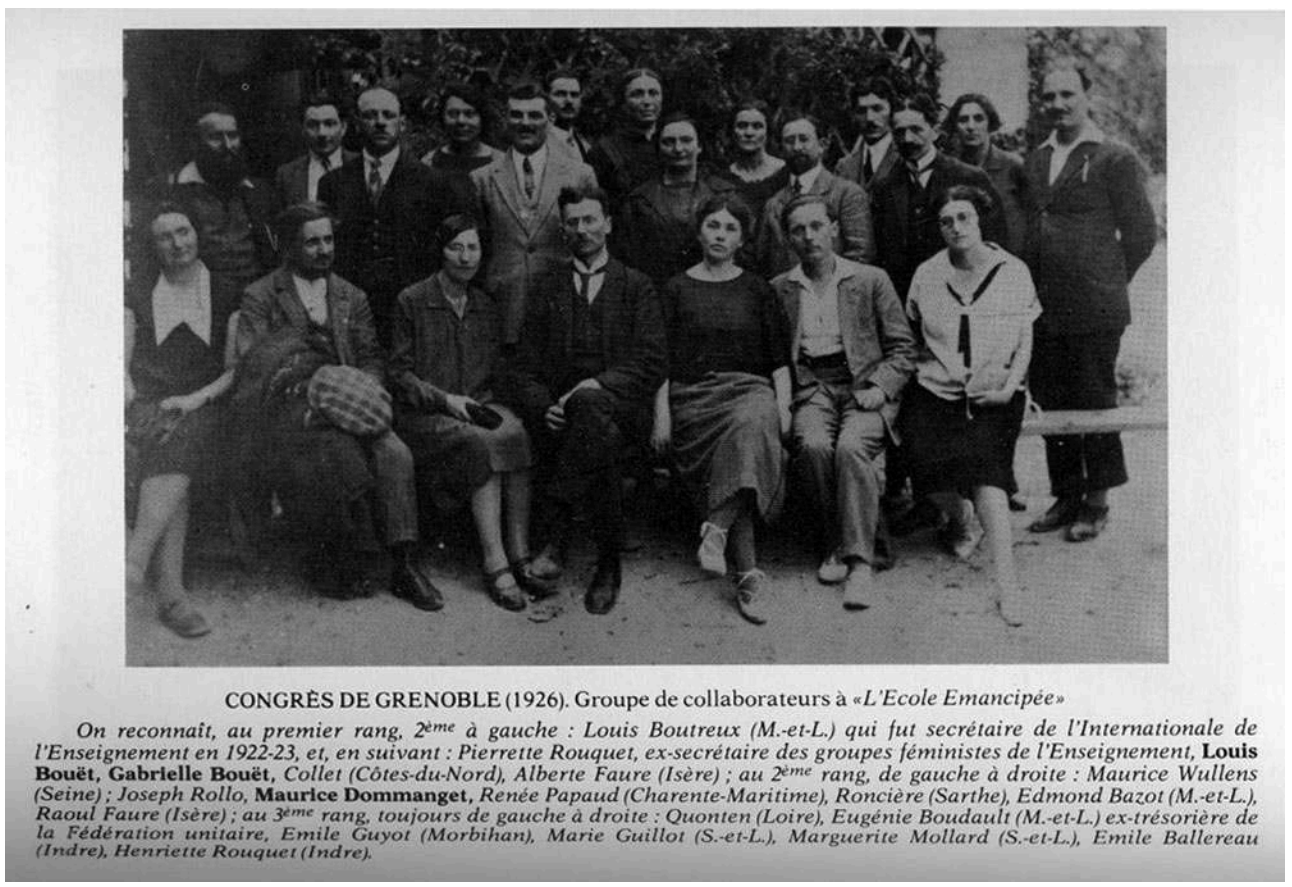




\section{Dommanget et sa femme}

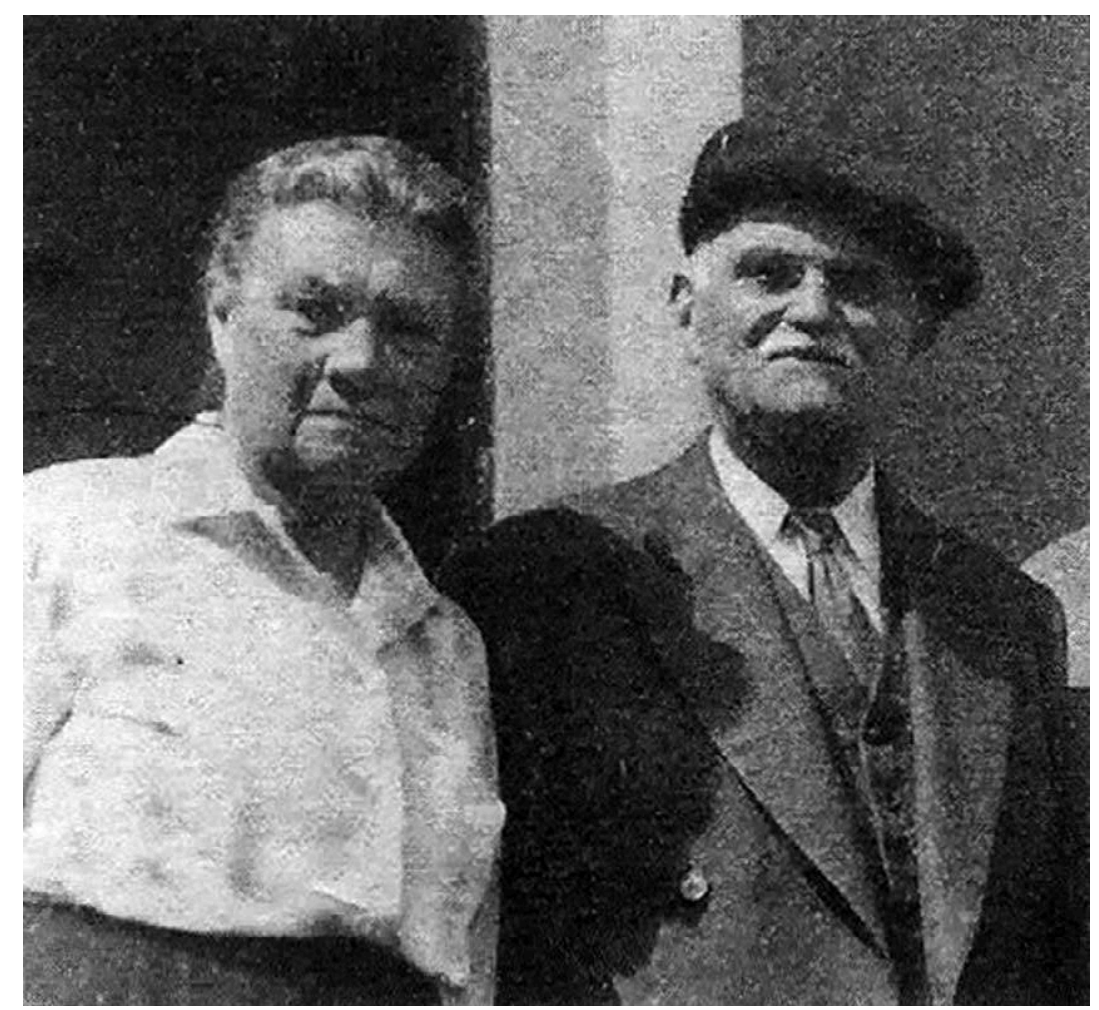

\section{4 . La première table des Annales Révolutionnaires}

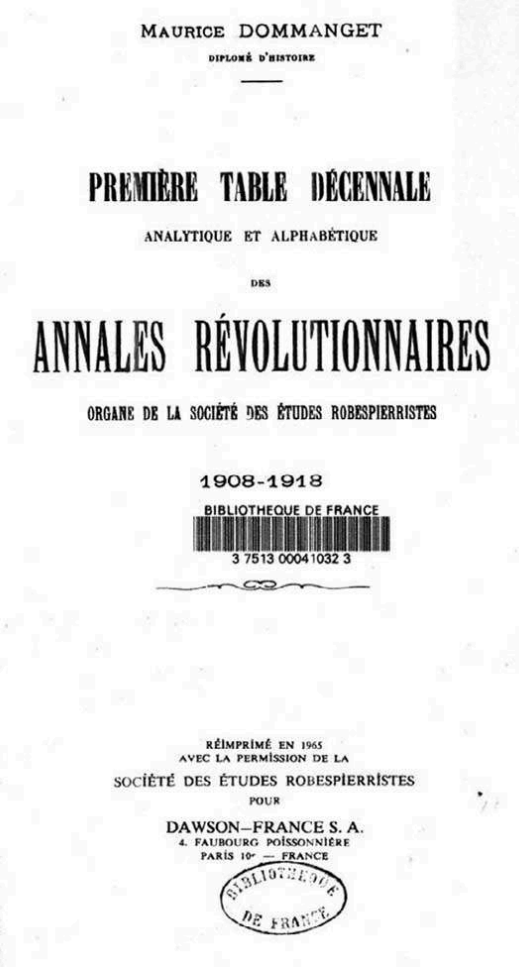

Annales historiques de la Révolution française, 353 | juillet-septembre 2008 


\section{L'entrée "Dommanget ", dans la première table des Annales Révolutionnaires}

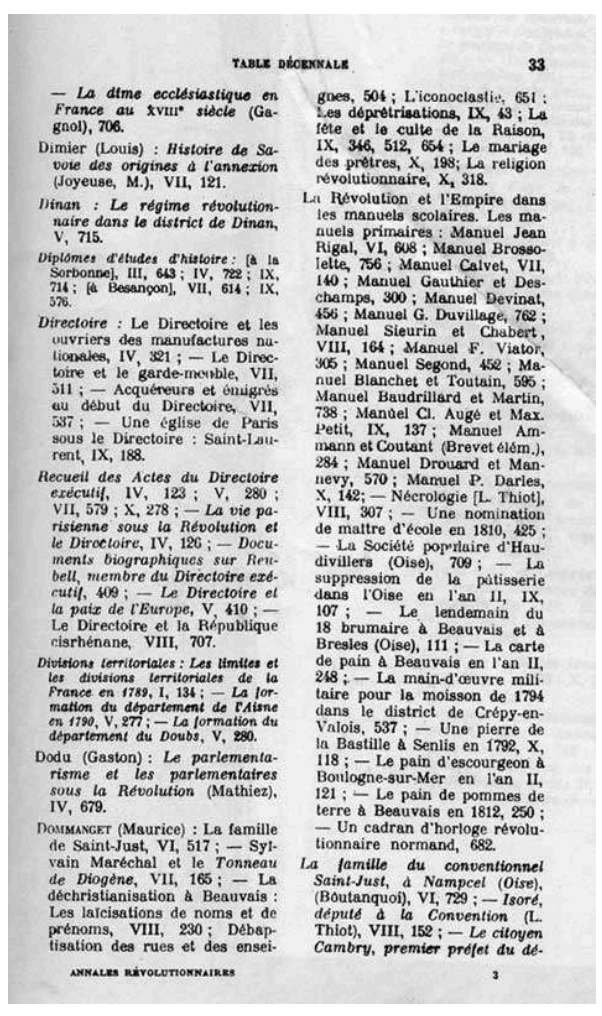

\section{L'entrée «Dommanget » dans la Table du centenaire}

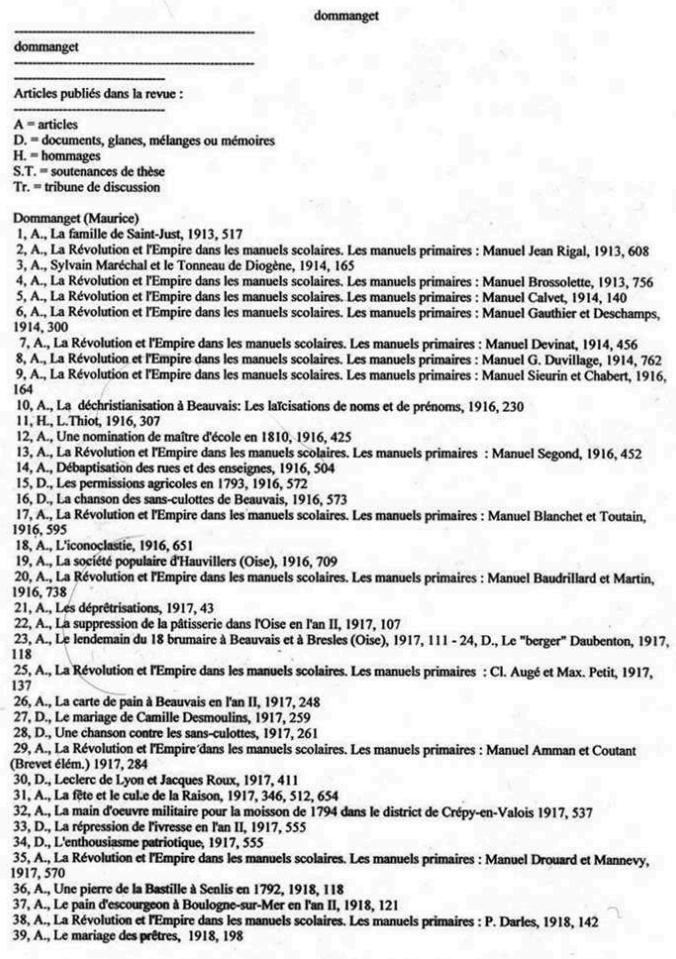




\section{NOTES}

1. «L'œuvre méconnue », Jean Rouch, Prolétaire en veston. Une approche de Maurice Dommanget, Les Monédières, 1984, p. 13.

2. À l'exception d'Ernest Labrousse, Robert Mandrou et surtout Albert Soboul qui assistent à ses obsèques. C'est Albert Soboul qui m'a alors chargé de rédiger l'hommage d'un jeune historien : «Maurice Dommanget (1888-1976)», AHRF, 227, 1977, p. 71-86. Je ne connaissais alors ni l'ampleur de son œuvre, ni la portée de ses engagements politiques et syndicaux.

3. L'éditorial, consacré à Albert Mathiez (1874-1932) et au colloque de Dijon (novembre 1974) dont il est le sujet central, ne mentionne pas la coïncidence avec le décès de Maurice Dommanget, qui n'est pas évoqué non plus dans les articles concernant le professeur.

4. "Maurice Dommanget", article cité, p. 71-73 et 84-86 ; surtout les actes du colloque de Beauvais (mai 1994), Maurice Dommanget (1888-1976), citoyen, pédagogue, historien, Compiègne, Archives départementales de l'Oise, 1996.

5. C'est le titre (heureux à notre sens) de l'ouvrage cité de Jean Rouch.

6. Voir la notice du Dictionnaire biographique du mouvement ouvrier français, le Maitron, tome 25, rédigée par Jean MAITRON - avec qui Dommanget partage bien des traits - et Claude PENNETIER.

7. Au moment des obsèques de Louise Michel, à laquelle il consacrera une publication, admirant son engagement politique et sa pédagogie révolutionnaire.

8. Erica J. MANUCCI, «La recherche de Dommanget sur Sylvain Maréchal», dans Maurice Dommanget..., op. cit., 1996, p. 215-229.

9. Dès 1907, à Montataire, dans une période d'affrontement entre l'État et le syndicalisme révolutionnaire. Voir Victor, Émile, Georges, Fernand et les autres... Regards sur le syndicalisme révolutionnaire, colloque de Nérac (novembre 2006), Les éditions d'Albret, 2007.

10. Il écrit dans La Franche-Comté socialiste pendant son service militaire à Montbéliard et assiste en uniforme à des réunions politiques.

11. Il tient une rubrique régulière dans le Travailleur de l'Oise, puis le Prolétaire de l'Oise et le FrancParleur de l'Oise, d'après Jean-Pierre BESSE, dans Maurice Dommanget..., op. cit., 1996, p. 21.

12. Vers 25 ans, il occupe déjà des responsabilités dans la revue pédagogique L'École émancipée (7), qu'il a contribué à fonder en 1910, portant comme devise des instituteurs qui la rédigent : "Instruisons-nous et armons-nous ». Il signe le manifeste avec son épouse, Eugénie et deux autres instituteurs.

13. Il adhère au parti socialiste après avoir obtenu le brevet supérieur, dès 1908 !

14. Julien DESACHY, dans Maurice Dommanget..., op. cit., 1996, p. 24.

15. Les archives seront détruites par la suite dans un bombardement.

16. Qu'il a contribué à fonder en 1910, à 22 ans.

17. Entre 1910 et 1939, 106 articles de Maurice Dommanget traitent de la Révolution française dans la revue L'École émancipée, Laurence BENICHOU, dans Maurice Dommanget..., op. cit., 1996, p. 89.

18. Dictionnaire Maitron, tome 25 , notice de Jean MAÎTRON et Claude PENNETIER.

19. «Dommanget, qu'on sent plein de maîtrise de soi, de profonde conviction, de largeur d'esprit ", L'Émancipation, 1926.

20. Lettre de Simone Collinet, du 17 octobre 1979, Jean-Louis Rouch, op. cit., p. 209.

21. En août 1929 se place l'épisode d'un voyage manqué en URss pour cause de faux papiers non parvenus à temps.

22. Il fait partie en 1930 de la «minorité oppositionnelle révolutionnaire », puis de l'opposition unitaire, aux côtés d'Alfred Rosmer, Loïc LE BRAS, dans Maurice Dommanget, op. cit., 1996, p. 74. Il rencontre avec 7 camarades Léon Trotsky à Domène (Isère), sans accord fi nal sur les orientations politiques et syndicales. 
23. Il s'oppose aux "purges » des années 1930 en URSS. Il est qualifié dans un rapport de 1937 d'«élément d'un sectarisme borné »!

24. Contrôleur des céréales à Beauvais entre 1940 et 1945, il a eu plus de chance que ses camarades syndiqués Louis Bouët (interné) et Jean Rollo (déporté).

25. L'attitude de Maurice Dommanget pendant la guerre a été diversement interprétée, en raison de son pacifi sme foncier et de son internationalisme. Il n'a pas été inquiété au lendemain de la Libération, où il retrouve tout naturellement sa place dans la Société des études robespierristes.

26. On peut consulter les Achives Dommanget, à l'Institut français d'histoire sociale, 60 rue des Francs Bourgeois, Denise FAUVEL-ROUIF, dans Maurice Dommanget, op. cit., 1996, p. 243-248.

27. Albert Soboul, Jean Deprun et Roland Desné.

28. Il lui consacre son diplôme d'études supérieures et un ouvrage qu'il revendique comme son meilleur livre.

29. Il préparait à 88 ans un recueil sur Jean Jaurès, qu'il admire, malgré ses tendances déistes.

30. Auteur du tome 5 de L'Histoire socialiste de la Révolution française, un des fondateurs du Parti ouvrier français avec Jules Guesde et Paul Lafargue, en 1889 ; auteur (entre autres) d'études sur le 1 er mai et sur Babeuf, et témoin de la libération d'Auguste Blanqui en 1879.

31. Maurice DOMMANGET, article dans L'École émancipée de 1932, au moment du décès de Mathiez.

32. La Révolution dans le canton de Neuilly-saint-Front (Aisne) : étude historique documentaire, Beauvais, Imprimerie Prévot, 1913.

33. Annales révolutionnaires, 1913, p. 426. Dommanget sera pourtant unanimement salué comme le modèle de l'historien « local»!

34. « La famille de Saint-Just », Annales révolutionnaires, 1913, p. 517.

35. « Le manuel Jean Rigal », Annales révolutionnaires, 1913, p. 608 ; « Le manuel Brossolette », id., p. 756.

36. La première assemblée est tenue en 1908.

37. Mais seulement 14 reconnus dans l'assistance (et 20 excusés). L'assemblée s'est tenue le 19 avril 1914

38. Dernier manuel analysé : P. Darles, Annales révolutionnaires, 1918, p. 142.

39. Annales révolutionnaires, 1914, p. 141. En 1917 il conclut une rubrique comparable ainsi : «Le même refrain dans la même chanson ". Il déplore les mythes positifs autour de Mirabeau et Danton.

40. Annales révolutionnaires, 1917, p. 139.

41. À propos du manuel Darles, Annales révolutionnaires citées, p. 143.

42. En compagnie de Gustave Rouanet.

43. Rapport du secrétaire de l'Assemblée générale de 1916.

44. Id., 1916, p. 709-711.

45. Albert MATHIEZ, Le bolchévisme et le jacobinisme, Paris, 1920.

46. Il est désigné ainsi dans la rubrique "Nos amis mobilisés ", Annales révolutionnaires, 1917, p. 164.

47. Annales révolutionnaires, 1917.

48. Époque de son adhésion au parti communiste à Tours en 1920, de sa démission en 1929 et de son mandat syndical auprès de le Fédération unifiée de l'enseignement au temps du front populaire.

49. En 1930, il doit faire une communication à l'assemblée générale, mais se plaint de n'avoir pas reçu la convocation.

50. Ils ont œuvré chacun de leur côté pour la reconnaissance des droits syndicaux des professeurs : Mathiez à Caen et Besançon, Dommanget à Besançon et Montataire.

51. Parmi les lectures proposées aux enfants fi gurent des textes de Mathiez, Aulard, Jaurès et... Dommanget : Jean-Louis ROUCH, op. cit., p. 37. 
52. Avec Henri Sée, professeur à Rennes, dont les engagements historiques aux côtés de Mathiez et de Dommanget mériteraient une étude spécifi que.

53. L'un des historiens qui a le plus compté pour Maurice Dommanget, autour des recherches sur le 1er mai, Paul Lafargue et la Commune de 1871.

54. «Il le quitta [le parti communiste] comme tant d'autres, obéissant au seul dictamen de sa conscience, conservant ses convictions ». "Mathiez, professeur et historien de la Révolution française ", par Maurice DOMMANGET, École Émancipée, avril 1932, page 220. On ne saurait mieux marquer le parallèle entre les deux engagements.

55. Maurice DOMMANGET, Le Symbolisme et le prosélytisme révolutionnaires à Beauvais et dans l'Oise (1790-1801), Beauvais, Imprimerie Librairie Prévot, 1932 ; précédé par Albert MATHIEZ, Les origines des cultes révolutionnaires, Paris, 1904.

56. Albert MATHIEZ, La vie chère et le mouvement social sous la Terreur, Paris, 1927 ; Maurice DOMMANGET, Les Enragés contre la vie chère..., Paris, Spartacus, 1948.

57. Jean-Louis ROUCH, op. cit., p. 134.

58. Jean-Louis ROUCH, op. cit., p. 132.

59. Le Magazine littéraire, 1970.

60. Déclaration de l'assemblée générale de la SER en 1917 : «Elle souhaite ardemment que la révolution russe trouve pour la diriger des Robespierre et des Saint-Just ", Annales révolutionnaires, 1917, p. 417.

61. Dommanget fait preuve en permanence d'une laïcité militante. Il mène constamment la lutte pour la « libre pensée » : Joseph BERNY, « Maurice Dommanget et la Libre Pensée », dans Maurice Dommanget, op. cit., 1996, p. 25-30.

62. Claude MAZAURIC souligne le fossé entre l'instituteur autodidacte et tel ancien élève de Normale supérieure, dans Maurice Dommanget, op. cit., 1996, p. 122.

63. Entretien donné au Magazine Littéraire, 46, novembre 1970, p. 36.

64. Michel vovelle, conclusion de Maurice Dommanget, op. cit. de Beauvais, 1996, p. 250.

65. Par exemple sur la question de la Marseillaise et de l'Internationale au moment du front Populaire : De la Marseillaise de Rouget de Lisle à l'Internationale d'Eugène Pottier, Paris, 1938.

66. Il reste à dépouiller le fonds Dommanget à l'Institut français d'histoire sociale pour trouver dans sa correspondance d'autres relations avec cette Société.

67. L'École émancipée, décembre 1932, en matière d'épitaphe.

68. Tables du centenaire, Claude COQUARD (dir.) , Paris, SER, 2008.

69. On ne parle pas ici des notes de lecture sur ses ouvrages traitant de la Révolution ou de sujets proches recensés dans d'autres revues.

70. 674 références à des contributions et 25 ouvrages ayant fait l'objet d'une note de lecture, entre 1908 et 1979.

71. 110 références, entre 1926 et 1992.

72. 43 références, entre 1908 et 1910.

73. 97 références, entre 1932 et 1954.

74. 92 références, entre 1910 et 1931.

75. 77 références, entre 1910 et 1965.

76. 50 références, entre 1910 et 1953.

77. 109 références, entre 1926 et 1985.

78. 87 références, entre 1951 et 1997.

79. AHRF, 1953, p. 378.

80. AHRF, 1967, p. 545.

81. Exemple : L'Enseignement, l'enfance, et la culture sous la Commune, Paris, Librairie de l'Étoile, 1964. 
82. «Si je ne travaille pas je m'ennuie. Qu'est-ce que je ferais si je n'avais pas mes livres ?", correspondance citée dans Jean-Louis ROUCH, op. cit., p. 87.

83. Ces cultes ou cette « religion révolutionnaire » représentent environ 25 contributions.

84. Annales révolutionnaires, 1918, p. 364.

85. Id., p. 706.

86. Annales révolutionnaires, 1917, p. 654 ; Serge BIANCHI, «Maurice Dommanget et les curés rouges ", colloque de Beauvais Maurice Dommanget (1994), Beauvais, Archives départementales de l'Oise, p. 155-162.

87. Il les condamne avec force tout en hésitant sur la qualificatif : iconoclasme en titre (1916, p. 651), vandalisme dans la démonstration.

88. Michel VovelLe, « Maurice Dommanget et le problème religieux », dans Maurice Dommanget, op. cit., 1996, p. 149-154.

89. Du pain en particulier (carte en 1917, p. 248 ; d'escourgeon en 1918, p. 121).

90. Pour les bacchanales (1924, p. 507), mises au point de Jacques BERNET et de Jean-Marc MORICEAU, Histoire et sociétés rurales, Nos 9 et 10, 1998.

91. Les permissions agricoles en 1916, p. 572.

92. Au total, une vingtaine de contributions échelonnées entre 1913 et 1968.

93. Une vingtaine de contributions également.

94. Au même titre que les sociétés populaires.

95. Babeuf et la conjuration des Égaux, Paris, Librairie de l'humanité, 1922 ; « Structure et méthode de la conjuration des Égaux ", Annales révolutionnaires, 1922, p. 177, 281 ; Babeuf, pages choisies, Paris, Armand Colin, 1935 ; soit une douzaine de références.

96. Victor DALINE, Gracchus Babeuf à la veille et pendant la Grande révolution française, Moscou, 1963, p. 427 (en russe). C'est la traduction des œuvres de Dommanget qui introduit les études babouvistes en Chine à partir de 1937.

97. Son dernier article dans les Annales en 1968 porte sur Saint-Just, 55 ans après son premier ! AHRF, p. 111.

98. Annales révolutionnaires, « Robespierre au collège », 1920, p. 153 ; « Robespierre et les cultes », 1924, p.193 ; AHRF, « Robespierre et le mariage des prêtres », 1951, p. 85.

99. Il souscrit en 1919, à Morvillers, pour le monument à Robespierre.

100. Petites capitales pour les auteurs des articles, caractères romains pour les auteurs qui font l'objet d'une note de lecture, italiques pour un sujet, objet ou lieu.

101. Les glanes et les documents sont en corps réduit par rapport aux articles de fond.

102. P. 62-74.

103. Annales révolutionnaires, 1920 , p. 33-34.

104. Qui se tenait alors dans la salle de l'École des hautes études en sciences sociales, avant la salle de la Sorbonne.

105. Annales révolutionnaires, 1920 , p. 556.

106. Annales révolutionnaires, 1918, p. 562.

107. Annales historiques, 1958, p. 7.

108. Id., p. 26.

109. AHRF, 1964, p. 253.

110. Robert LEGRAND défi nit ainsi son ami : «Un travailleur simple et droit, chaleureux et passionné », dans Maurice Dommanget, op. cit., 1996, p. 16.

111. À propos des « curés rouges ", dans Maurice Dommanget, op. cit., de Beauvais, 1996, p. 160-161.

112. Par exemple sur Lénine, un "jacobin s'appuyant sur la classe ouvrière"; ou sur Le Manifeste des Égaux : "Toutes les couleurs de l'arc-en-ciel du socialisme intransigeant et révolutionnaire et y compris l'anarchisme communiste sont plus ou moins empruntées au Manifeste des Égaux ", Babeuf et la conjuration des Égaux, Spartacus, 1923, réed. 1989, p. 80. 
113. Autre exemple : «Le Manifeste communiste est le meilleur manuel de la révolution prolétarienne et du socialisme ", La révolution de 1848 et le Drapeau rouge, Paris, Spartacus, 1948.

114. Babeuf et la Conjuration des Égaux, Paris, Spartacus, 1923, rééd. 1989, p. 80.

115. Annales révolutionnaires, 1917 , p. 526.

116. Michel VOVELLE, "Maurice Dommanget et le problème religieux », colloque cité, 1996, p. 149-154 ; Wolfgang GEIGER, "Les cultes de la Raison. Phénoménologie d'une "religion civile" pendant la Révolution, à partir des recherches de Dommanget », id., p. 163-170.

117. 1917, p. 652.

118. Serge BIANCHI, «Le "vandalisme révolutionnaire" et la politique artistique de la Convention au temps des "terreurs" : essai de bilan raisonné ", dans Michel BIARD (dir.), Les politiques de la Terreur 1793-1794, Rennes, Presses Universitaires de Rennes, Rennes, 2008 (actes du colloque de Rouen, 2007), p. 403-419.

119. Annales révolutionnaires, 1918 , p. 326.

120. « Maurice Dommanget, historien local », dans Maurice Dommanget, op. cit., p. 193-199.

121. «Un ouvrage pionnier », selon Jacques BERNET, dans Maurice Dommanget, op. cit., p. 193.

122. Voir les travaux de Jacques BERNET et de Serge BIANCHI, La déchristianisation dans le district de Corbeil 1793-1797, Millau, Mémoires de la SHACEH, 1990.

123. Maurice Dommanget, op. cit., p. 199.

124. Patrick GARCIA, Le Bicentenaire de la Révolution française, pratiques sociales d'une commémoration, Paris, CNRS-éditions, 2000.

125. Annales révolutionnaires, 1913, p. 427, pour le Brossolette, Histoire de France, cours moyen, Delagrave.

126. Annales révolutionnaires, 1914 , p. 140, à propos du Calvet.

127. Id., p. 726 : «Un mot qui n'est pas expliqué à une leçon, c'est un grain de poison dans un morceau de pain ».

128. Annales révolutionnaires, 1918 , p. 143 : « Aussi bien que savoir, douter a son prix ».

129. Annales révolutionnaires, 1917, p. 284.

130. Id., p. 139 ; « Le peuple n'est qu'une abstraction pour nos auteurs de manuels », p. 570.

131. Surtout quand on voit la faiblesse actuelle de notre implantation chez les instituteurs.

132. La Nouvelle histoire de France, dont la préface proclame : «Enfant, étudie cette petite histoire de ton pays. Elle n'a pas oublié les paysans, les ouvriers d'autrefois qui ont peiné, qui ont souffert ». Jean-Louis Rouch, op. cit., p. 46. Cela ne l'empêche pas d'avoir un différend avec Freinet, sur la question des manuels.

133. En témoignent les ouvrages sur les grands pédagogues comme Pestalozzi, Francesco Ferrer ou Paul Robin : Les grands socialistes et l'éducation de Platon à Lénine, Paris, Armand Colin, 1972.

134. Annales révolutionnaires, 1917, p. 284. Voir Janine SEUX, « Maurice Dommanget ou l'école de la vie », colloque cité, 1996, p. 41-48.

135. Autour de 430 exemplaires par numéro.

136. « Ni Dieu, ni César, ni Tribun», affi rme-t-il dans L'École Émancipée du 28 juin 1936 : JeanLouis ROUCH, op. cit., p. 70.

137. AHRF, 1958, p. 26-27.

138. $A H R F, 1964$, p. 264

139. L'orateur : «Il a de la flamme et de sa bouche au menton carré sortent des paroles qui exaltent » : Jean RABAUT, Tout est possible, p. 61.

140. AHRF, 1958, p. 27. 


\section{RÉSUMÉS}

Maurice Dommanget a joué un rôle essentiel au sein de la Société des études robespierristes et dans la rédaction des Annales révolutionnaires, puis des AHRF, de 1913 à 1964 . Très proche d'Albert Mathiez, cet instituteur de base, militant syndicaliste, socialiste puis communiste (jusqu'en 1929), antimilitariste, anime la SER jusqu'en 1926 et après sa retraite en 1948. Il est l'un des rédacteurs les plus assidus des Annales, avec plus de 140 contributions, l'auteur de la première Table décennale en 1919, l'historien de la revue en 1958. Il lance un Appel en 1964 pour élargir l'audience des $A H R F$ et poursuivre la mission de diffusion de l'histoire scientifique de la Révolution française, en rappelant le sens et les enjeux de la création de la SER. Historien des contestations sociales, politiques et religieuses, pédagogue de la « lutte des classes ", pionnier de l'histoire locale, Maurice Dommanget, "prolétaire en veston », mérite un hommage particulier au moment de la commémoration du centenaire d'une société et d'une revue auxquels il a tant donné.

Maurice Dommanget played an essential role in the Société des études robespierristes, and in the writing of the Annales révolutionnaires, then the AHRF from 1913 to 1964. Close to Albert Mathiez, he was essentially a primary school teacher, militant union member, socialist, then communist (until 1929), anti-militarist, who animated the SER until 1926, and afterhis retirement in 1948. He was among the most assiduous editors of the Annales, with more than 140 contributions to his name; the author of the first table decennale in 1919; the historian of the journal in 1958. He issued an appeal in 1964 to enlarge the public for the AHRF, and to pursue the mission of transmitting the scholarly history of the French Revolution by recalling the meaning and the critical issues involved for the creation of the SER. A historian of social conflict, political no less than religious, Maurice Dommanget, a « proletarian in a suit » deserves special recognition at the commemoration of the centennial of the organization, and of the journal for which he did so much.

\section{INDEX}

Mots-clés : Dommanget, Mathiez, Annales révolutionnaires, AHRF, Société des études robespierristes, militant, pédagogue, citoyen

\section{AUTEUR}

\section{SERGE BIANCHI}

Serge Bianchi, Université de Rennes 2, 26, allée des deux Cèdres, 91210 Draveil, sbianchi2@aol.com 